\title{
Type 2-High Severe Asthma with and without Bronchiectasis: A Prospective Observational Multicentre Study
}

\author{
Claudia Crimi (D) \\ Raffaele Campisi (iD \\ Santi Nolasco iD ${ }^{2}$ \\ Sebastian Ferri iD $^{3,4}$ \\ Giulia Cacopardo ${ }^{5}$ \\ Pietro Impellizzeri ${ }^{2}$ \\ Maria Provvidenza Pistorio ${ }^{2}$ \\ Evelina Fagone ${ }^{2}$ \\ Corrado Pelaia (iD ${ }^{6}$ \\ Enrico Heffler ${ }^{3,4}$ \\ Nunzio Crimi (iD) ${ }^{1,2}$ \\ 'Respiratory Medicine Unit, A.O.U. \\ Policlinico "G. Rodolico - San Marco", \\ Catania, Italy; ${ }^{2}$ Department of Clinical \\ and Experimental Medicine, University of \\ Catania, Catania, Italy; ${ }^{3}$ Personalized \\ Medicine, Asthma and Allergy - IRCCS \\ Humanitas Research Hospital, Rozzano, \\ Italy; ${ }^{4}$ Department of Biomedical \\ Sciences, Humanitas University, Pieve \\ Emanuele, MI, Italy; ${ }^{5}$ Respiratory \\ Intensive Care Unit, ARNAS Civico \\ General Hospital, Palermo, Italy; \\ ${ }^{6}$ Department of Medical and Surgical \\ Sciences, University "Magna Graecia”, \\ Catanzaro, Italy
}

Correspondence: Claudia Crimi Respiratory Medicine Unit, A.O.U. Policlinico "G. Rodolico - San Marco", Catania, Italy

Email dott.claudiacrimi@gmail.com
Introduction: Type 2-high severe asthma (T2-SA) is often associated with several comorbidities. To this extent, the coexistence of T2-SA and bronchiectasis (BE) is considered an emerging phenotype.

Methods: We performed a prospective observational multicentre study, including T2-SA patients. Chest HRCT confirmed the presence of BE. Data on exacerbations, pulmonary function, Asthma Control Test (ACT), chronic mucus hypersecretion $(\mathrm{CMH})$, chronic rhinosinusitis (CRS), oral corticosteroid (OCS) dosage, eosinophils in peripheral blood and FeNO were recorded. The Bhalla score was used for radiological assessment of T2-SA+BE patients and the Bronchiectasis Severity Index (BSI) was calculated.

Results: A total of 113 patients (mean age $55 \pm 11$ years, $59.3 \%$ female) were enrolled. Copresence of BE was confirmed in 50/113 (44.2\%) patients who identified the T2-SA+BE group. CRS and CRSwNP were more prevalent in T2-SA+BE vs T2-SA [respectively, $42 / 50$ (84\%) vs $37 / 63$ (58.7\%), $\mathrm{p}=0.004$ and $27 / 50$ (54\%) vs 27/63 (42.9\%), $\mathrm{p}=0.0165]$. Furthermore, T2-SA $+\mathrm{BE}$ patients reported more CMH compared to T2-SA [29/50 (58\%) vs 15/63 (23.8\%), p = $0.0004]$, were more frequently on chronic OCSs intake [28/50 (56\%) vs 22/63 (34.9\%), p = 0.0357 ] and experienced more exacerbations/year [10 (4-12) vs $6(4-12), \mathrm{p}=0.0487]$. In a multivariate logistic regression model, the presence of CRS, CMH and daily OCS intake were associated with BE presence with a 78\% (95\% CI: 69-88) accuracy. Median Bhalla score was 18.3 (16-20) (Mild radiological severity). Median BSI was $6(4-8)$ and only 6/50 (12\%) had a BSI score $\geq 9$. Significant inverse linear relationship between BSI and ACT $(r=-0.6095, p<$ 0.0001), $\mathrm{FEV}_{1} \%(\mathrm{r}=-0.3297, \mathrm{p}=0.0353)$ and $\mathrm{FEV}_{1} \mathrm{~mL}(\mathrm{r}=-0.4339, \mathrm{p}=0.0046)$ were found. Conclusion: Type 2 inflammation could have a causative role in BE development. Chest HRCT is mandatory when a diagnosis of T2-SA is made, especially in presence of CRS, $\mathrm{CMH}$ and chronic OCS intake. Early BE detection may be crucial to improve T2-SA patients' outcomes.

Keywords: type 2 inflammation, severe asthma, bronchiectasis, chest-CT scan, phenotype

\section{Introduction}

Severe asthma (SA) is a chronic respiratory disease characterized by bronchial inflammation and airway hyperresponsiveness, inciting daily symptoms and triggering unpredictable acute exacerbations. ${ }^{1,2}$ The prevalence of SA varies between $3.7 \%$ and $10 \%$ of the asthma framework, with a high burden on the health-care system. $^{3-5}$

Recent clinical and translational studies have enabled endotyping, allowing to dichotomize asthma according to levels of type 2 inflammation into "type 2 (T2)- 
high" and "T2-low" subtypes. Type 2 inflammation, mediated by cytokines such as interleukin-4 (IL-4), interleukin-5 (IL-5), and interleukin-13 (IL-13), occurs in approximately $70 \%$ of asthmatic patients. ${ }^{6}$ Moreover, several comorbidities are associated with type 2-high severe asthma (T2-SA), making their recognition and treatment crucial to improve disease control and outcomes. ${ }^{7,8}$

Recently, the coexistence of T2-SA and bronchiectasis (BE) has been considered an emerging phenotype of severe and difficult-to-treat asthma ${ }^{9,10}$ associated with a worse prognosis of the disease, ${ }^{11}$ representing one of the "treatable traits" highlighted in the latest asthma recommendation. ${ }^{4,12}$ Indeed, the prevalence of $\mathrm{BE}$ is significantly higher in SA (range 24-47\%) than in mild-tomoderate asthma $(3 \%) .{ }^{10,11,13}$

It has been hypothesized that the long-term eosinophilsmediated inflammatory damage, promoted by T2inflammation, induces tissue changes and airway remodeling, playing a primary role in the pathogenesis of $\mathrm{BE}^{14,15}$ Nevertheless, this pathogenetic mechanism has not been fully elucidated yet, and the clinical and radiological characteristics of this phenotype have not been clearly defined.

In this study we aimed to:

1) describe the clinical and functional differences of T2-SA patients with and without BE;

2) evaluate the radiological characteristics and clinical severity of $\mathrm{BE}$;

3) specifically assess the clinical impact of BE on T2SA according to their severity.

\section{Materials and Methods Study Design and Patient Population}

This prospective, observational, multicentre study was conducted in two severe asthma-dedicated outpatient services in Italy: (1) Respiratory Medicine Unit - A.O.U. Policlinico "G. Rodolico - San Marco", Catania; (2) Personalized Medicine, Asthma and Allergy, Humanitas Research Center IRCCS, Rozzano, Italy between December 2018 and December 2019. Consecutive patients with a T2-SA, defined by a blood eosinophils count $\geq 150$ cells/ $\mu \mathrm{L}$ and/or FeNO $\geq 20 \mathrm{ppb}$ and/or sputum eosinophils $\geq 2 \%$, and/or asthma clinically allergen-driven and/or need oral corticosteroids (OCS) for maintenance were included. $^{4}$ We excluded individuals with immunological deficits, allergic bronchopulmonary aspergillosis (ABPA), alpha-1-deficiency, vasculitis, cystic fibrosis, primary ciliary dyskinesia, T2-Low SA, and with previous pneumonia episodes, which could also have the presence of BE.

Asthma diagnosis was assessed and confirmed with a positive bronchial reversibility test (defined as a change in $\mathrm{FEV}_{1}$ of at least $12 \%$ and $200 \mathrm{~mL}$ from the prebronchodilator value, measured 15 minutes after the inhalation of salbutamol $400 \mathrm{mcg}) .{ }^{4}$ SA diagnosis was defined according to the European Respiratory Society/American Thoracic Society (ERS/ATS) guidelines. ${ }^{2}$ T2-SA assessment criteria were in agreement with Global Initiative for Asthma (GINA) 2019 report. $^{4}$

As a routine workup, serum IgA-IgG-IgM, IgG subclasses and lymphocyte typing were tested to assess immunological status and testing for allergic bronchopulmonary aspergillosis were performed as suggested by the European Respiratory Society guidelines on bronchiectasis. ${ }^{12}$ Sweat testing was performed only in patients showing diffuse bronchiectasis at the chest CT scan and with clinical history suspicious for cystic fibrosis. ${ }^{16}$

This study adhered to the Declaration of Helsinki and was approved by the "Catania 1" Ethics Committee of the A.O.U. Policlinico "G. Rodolico - San Marco" in Catania, Italy (Protocol Number 108/2018/PO). Written informed consent was obtained from all patients.

\section{Data Collection}

An established database of pertinent variables was created. Social and demographic data (age, sex, BMI), past medical history, smoking status, and asthmatic profiles [age at onset, sensitization to perennial aeroallergens, pulmonary function, blood analysis for eosinophils count, total IgE level, fractional exhaled nitric oxide (FeNO) value, asthma control test (ACT), sputum characteristic and microbiology, number of asthma exacerbations requiring oral or systemic corticosteroids treatment for at least 3 days and/ or emergency visits/hospitalizations in the last 12 months] were collected. Information about the ongoing treatments [inhaled corticosteroids (ICS), long-acting beta-agonists (LABAs), long-acting muscarinic receptor antagonists (LAMAs), leukotriene modifiers and oral corticosteroids] was also reported. Furthermore, available information about other comorbidities (nasal polyps, rhinosinusitis, GERD, obesity) was included. BE diagnosis was documented by HRCT scan. Severity of BE was evaluated from a clinical and radiological point of view, according to the 
Bronchiectasis Severity Index (BSI) and Bhalla score, respectively.

\section{Measurements}

\section{Asthma Exacerbations}

Severe asthma exacerbations were defined as disease worsening requiring hospitalization or $\geq 3$ days of treatment with systemic corticosteroids (or a doubling of prednisone equivalent dose if already on OCS). ${ }^{17}$

\section{Pulmonary Function}

Pulmonary function tests were performed according to ERS/ATS guidelines. Forced Vital Capacity (FVC) and Forced Expiratory Volume in 1 second $\left(\mathrm{FEV}_{1}\right)$ were measured using a spirometer (Sensormedics, Milan, Italy). The best value of three consecutive manoeuvres was expressed as the percentage of the normal value. Bronchodilator reversibility testing was performed. ${ }^{18}$

\section{Asthma Control Test}

Levels of asthma control were assessed using the $\mathrm{ACT},{ }^{19,20}$ a short, simple five-point scoring system questionnaire eliciting a patient's perception of symptoms control. An overall score $\geq 20$ means well-controlled asthma, whereas a score $\leq 19$ reflects poor asthma control.

\section{FeNO Measurements}

The fraction of exhaled nitric oxide was performed according to ERS/ATS guidelines and was measured using the Niox ${ }^{\circledR}$ device. FeNO levels were considered normal if FeNO $<25$ parts per billion (ppb) and elevated if FeNO $>50 \mathrm{ppb}$, as recommended. ${ }^{21}$

\section{Chronic Mucus Hypersecretion}

Symptoms of chronic mucus hypersecretion (CMH), defined as cough and sputum production on most days for at least three months a year for at least two consecutive years, were recorded. Mucus was defined as mucous if its colour was white to light yellow, muco-purulent if yellow to light green and purulent if green or dark green. ${ }^{22}$

\section{Chronic Rhinosinusitis with Nasal Polyps}

The diagnosis of chronic rhinosinusitis (CRS), according to the European position paper on rhinosinusitis and nasal polyps (EPOS) $2020,{ }^{23}$ was formulated on symptoms (nasal congestion, nasal discharge, facial pain/pressure, reduction/loss of smell), present for a minimum of 12 weeks. In case of objective confirmation of the presence of polyps with sinus computed tomography scan or nasal endoscopy, the diagnosis of chronic rhinosinusitis with nasal polyps (CRSwNP) was made. ${ }^{23}$

\section{Radiological Assessment of Bronchiectasis}

All patients underwent High-Resolution Chest Tomography (HRCT), performed during stable conditions. Images were obtained during a complete inspiration at 1-mm collimation and $10-\mathrm{mm}$ intervals, from lung apices to bases, in the supine position. Two independent radiologists, blinded to research findings, evaluated the images. The presence of BE was reported according to the following radiological criteria: 1) internal diameter of the bronchus greater than that of the adjacent pulmonary artery; 2) lack of tapering of the bronchial lumen toward the periphery. Bronchiectasis only visible in a single pulmonary segment was not considered. $^{24-26}$

The radiological severity and extension were assessed with the Bhalla score. ${ }^{27}$ The Bhalla score range goes from 0 to 25 , with a lower score indicating more radiologically severe bronchiectasis. The total was calculated by scoring each of the nine categories and then subtracting from 25 . This score was subdivided into mild (16-25), moderate (9-15) and severe (0-8).

We also assessed the number of lobes (the lingula was considered a separate lobe) with bronchiectasis and their prevalent localization. The BE distribution patterns were categorized as central, peripheral or mixed. The type of $\mathrm{BE}$ was reported according to the Reid classification. ${ }^{28}$

\section{Severity Assessment of Bronchiectasis}

The Bronchiectasis Severity Index (BSI) is a multidimensional scoring system that has been recently introduced to better understand the disease. The BSI has shown to be an excellent predictor of mortality and hospital admissions and a predictive index of exacerbations. It was calculated in patients affected by bronchiectasis, combining body mass index (BMI), $\mathrm{FEV}_{1}$ $\%$ predicted, hospital admission in the past two years, number of exacerbations in the previous year, modified Medical Research Council (mMRC) breathlessness score, radiological severity ( $>3$ lobes involved or cystic changes), presence of pseudomonas and chronic bacteria colonization.

Microbiology testing was performed on patients' spontaneous early morning sputum samples and analysed for bacterial, fungal and mycobacterial cultures. We collected sputum at least on 2 occasions, with a minimum of 3 months for 1 year, to assess the possible presence of chronic colonization. ${ }^{29,30}$ Subjects unable to provide sputum samples due to the absence of a productive cough 
were classified as not having chronic infection for analysis purposes. According to the BSI overall score (range from 0 to 26), bronchiectasis was defined as mild (BSI $=0-4$ points), moderate (BSI $=5-8$ points), or severe (BSI $\geq 9$ points). ${ }^{31}$

\section{Statistical Analysis}

Statistical analysis and figures were generated using Prism version 9.0.2 (GraphPad Software Inc). Categorical variables were stated as numbers (n) and percentages (\%). Normally distributed continuous variables were expressed as mean \pm standard deviation (SD). Median and interquartile range (IQR) were used in case of continuous nonparametric variables. Agreement between the radiologists was estimated using the kappa Intraclass Correlation Coefficient (with 95\% CIs). Fisher exact or Chi-Square tests were used for comparisons of categorical variables. The normality of data distribution was checked using the Anderson-Darling and Kolmogorov-Smirnov tests. MannWhitney $U$-test or Student's $t$-test were used for continuous data, respectively, when appropriate. Linear regressions analysis with Spearman's rank coefficient were used to evaluate the correlation between variables. Multivariable analyses were performed using a logistic regression model (with 95\% CIs). Variable selection using subsampling methodology was conducted to construct the optimal model. A p-value $<0.05$ (two-sided) was considered statistically significant.

\section{Results}

Characteristics and Differences Between Type-2 High Severe Asthma Patients with and without Bronchiectasis

A total of 195 SA patients were screened. Thirteen did not sign the informed consent. Sixty-seven were excluded after the clinical assessment. One-hundred-fifteen underwent a high-resolution chest CT scan. Two patients were suspected of cystic fibrosis. Five patients had a supportive history of primary cilia dyskinesia and were addressed to centers with expertise in the field to confirm or exclude the diagnosis. One patient resulted positive to the genetic tests for cystic fibrosis and one showed defects in cilia movements compatible with primary cilia dyskinesia (Figure 1). One-hundred thirteen patients met the inclusion criteria and were included in the analysis. The mean age of the total cohort was $55 \pm 11$ years. Mean age at onset was 32.3 \pm 15.5 years. The majority of patients were female (67/
$113,59.3 \%)$. All patients $(113 / 113,100 \%)$ were prescribed high-dose ICS/LABA and 69\% (78/113) were on add-on therapy with LAMA. Eighty-two (72.6\%) had positive skin prick tests. Median eosinophil count in the peripheral blood was 500 (340-900) cells $/ \mu \mathrm{L}$, with $82.3 \%(93 / 113)$ showing $\geq 300$ eosinophils/ $\mu \mathrm{L}$. Fifty out of $113(44.2 \%)$ showed co-presence of bronchiectasis at chest HRCT and were identified as T2-SA+BE group. Baseline and clinical characteristics of patients of each group (T2-SA alone and $\mathrm{T} 2-\mathrm{SA}+\mathrm{BE}$ ) are reported in Table 1. No statistically significant differences in terms of ACT, pre-bronchodilator $\mathrm{FEV}_{1} \%, \mathrm{FEV}_{1} \mathrm{~mL}, \mathrm{FEV}_{1} / \mathrm{FVC} \%$, total $\mathrm{IgE}, \mathrm{FeNO}$ and blood eosinophil were noted between the groups. Chronic rhinosinusitis (CRS) and chronic rhinosinusitis with nasal polyps (CRSwNP) were significantly more prevalent in T2-SA+BE patients vs T2-SA without BE [respectively, $42 / 50(84 \%)$ vs $37 / 63(58.7 \%), p=0.004$ and $27 / 50(54 \%)$ vs $27 / 63(42.9 \%), p=0.0165]$ (Figure $2 A$ and B). Twentynine out of $50(58 \%)$ in the T2-SA+BE group, compared to $15 / 63(23.8 \%)$ T2-SA alone patients $(\mathrm{p}=0.0004)$, reported $\mathrm{CMH}$, in particular of muco-purulent [18/50 $(36 \%)$ vs $10 / 63(15.9 \%), p=0.0167]$ and purulent type [8/50 (16\%) vs $1 / 63(1.6 \%), p=0.01]$ (Figure 2C). A greater proportion of patients with bronchiectasis was on maintenance OCS [28/50 (56\%) vs 22/63 (34.9\%), p = 0.0357] (Figure 2D). Furthermore, T2-SA+BE patients reported a higher number of asthma exacerbations in the previous year [10 (4-12) vs $6(4-12), p=0.0487]$ (Figure 2E).

A multivariate logistic analysis was conducted based on the differences between the groups, and the optimal model included the following predictors that are strongly associated with comorbid BE in T2-SA: the presence of CRS, chronic sputum production, and daily OCS intake. The resulting accuracy of this model using the area under the curve (AUC) of the receiver operating characteristics (ROC) was $78 \%$ (95\% CI: 69-88), with a specificity of $76 \%$ and a sensibility of $74 \%$ (Figure $2 \mathrm{~F}$ ).

\section{Radiological Features of Bronchiectasis in Type-2 High Severe Asthma}

HRCT chest features of T2-SA+BE patients are listed in Table 2. The kappa coefficient between the chest radiologists was $0.92(95 \%$ CI: $0.84-0.99)$ for assessing the Bhalla score [median 18.3 (16-20)]. All the patients had mild $(38 / 50,76 \%)$ or moderate $(12 / 50,24 \%)$ bronchiectasis, in terms of radiological severity, with a median of 3 


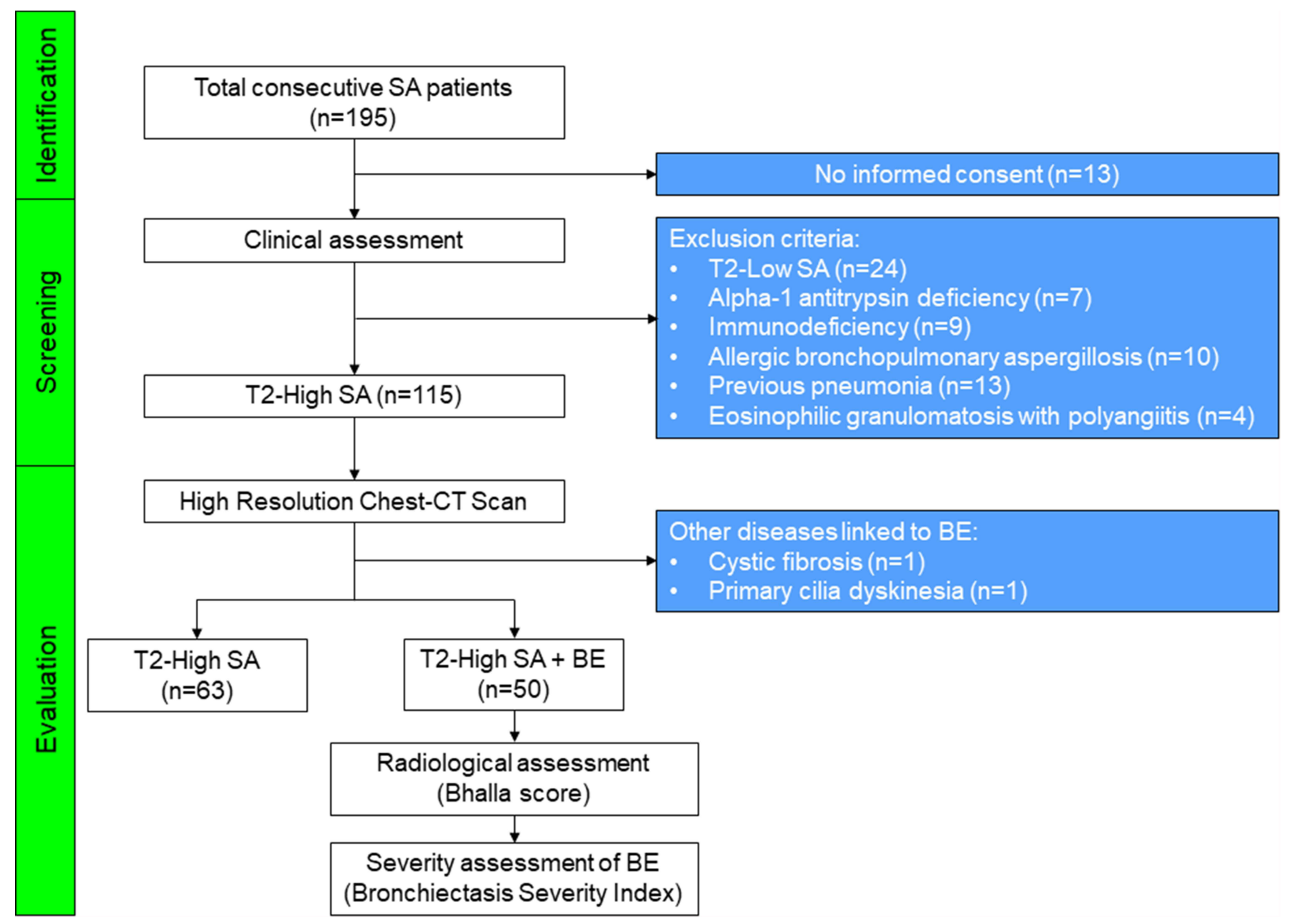

Figure I Study flow chart.

Abbreviations: SA, severe asthma; BE, bronchiectasis.

(2-4) lobes affected and predominantly periphery (72\%) and lower fields involvement (Figure 3A and B). Bhalla sub-items prevalence across $\mathrm{T} 2-\mathrm{SA}+\mathrm{BE}$ is presented in Figure 4.

No significant correlation was found between Bhalla score and years of asthma, asthma exacerbations, ACT score, $\mathrm{FEV}_{1} \%, \mathrm{FEV}_{1} / \mathrm{FVC} \%$, peripheral blood eosinophil count, total IgE, Vitamin D, Alpha-1 antitrypsin and FeNO.

\section{Clinical Impact of Bronchiectasis in Type-2 High Severe Asthma According to BSI}

In T2-SA+BE patients, the median BSI was 6 (4-8) (Table 2). Only 6 out of $50(12 \%)$ had a BSI score $\geq 9$, indicative of clinically severe bronchiectasis. Sputum microbiological examination showed chronic Pseudomonas aeruginosa colonization in 5 patients $(10 \%)$ and chronic
Stenotrophomonas maltophilia colonization in 1 patient (2\%) out of 50 .

We found a significant inverse linear relationship between BSI and ACT score (Figure 5A; $\mathrm{r}=-0.6095$, $\mathrm{p}<0.0001$ ), $\mathrm{FEV}_{1} \%$ (Figure $5 \mathrm{~B} ; \mathrm{r}=-0.3297, \mathrm{p}=$ 0.0353) and $\mathrm{FEV}_{1} \mathrm{~mL}$ (Figure $5 \mathrm{C} ; \mathrm{r}=-0.4339, \mathrm{p}=$ 0.0046 ) respectively. No significant correlation was found between BSI score and duration of asthma, asthma exacerbations, $\mathrm{FEV}_{1} / \mathrm{FVC} \%$, peripheral blood eosinophil count, total IgE, Vitamin D, alpha-1 antitrypsin and FeNO.

\section{Discussion}

In the present prospective observational study, we compared the clinical and functional differences of T2-SA patients with and without BE. We reported novel findings assessing the clinical and radiological characteristics of patients with T2-SA and coexisting BE, using the BSI and Bhalla score. ${ }^{27}$ The prevalence of BE in our cohort 
Table I Clinical and Functional Characteristic of Type-2 High Severe Asthma Patients with and without Bronchiectasis

\begin{tabular}{|c|c|c|c|c|c|}
\hline \multicolumn{3}{|l|}{ Total Population, $\mathrm{n}=1 \mathrm{I} 3$} & T2-SA, $n=63$ & T2-SA+BE $n=50$ & $\mathbf{p}$ \\
\hline \multicolumn{2}{|l|}{ Age, years, mean (SD) } & $55(\mathrm{II})$ & $54.9(11.4)$ & 55.5 (II.7) & 0.8107 \\
\hline \multicolumn{2}{|l|}{ Age at onset, years, mean (SD) } & $32.3(15.5)$ & $31.8(16.2)$ & $33(14.7)$ & 0.6792 \\
\hline \multicolumn{2}{|l|}{ Female, n (\%) } & $67(59.3)$ & $39(61.9)$ & $28(56)$ & 0.5667 \\
\hline \multicolumn{2}{|l|}{ BMI, mean (SD) } & $26.1(4.1)$ & $26(3.9)$ & $26(4.5)$ & 0.8330 \\
\hline \multicolumn{2}{|l|}{ Obese (BMI $\geq 30)$, n (\%) } & $23(20.4)$ & $12(19)$ & II (22) & 0.8149 \\
\hline \multicolumn{2}{|l|}{ Positive Skin Prick Tests, n (\%) } & $82(72.6)$ & $45(71.4)$ & $37(74)$ & 0.8336 \\
\hline \multirow[t]{3}{*}{ Smoking history, n (\%) } & No & $86(76.1)$ & $49(77.8)$ & $37(74)$ & 0.6629 \\
\hline & Current & $9(8)$ & $4(6.3)$ & $5(10)$ & 0.5058 \\
\hline & Ex-smoker & $18(15.9)$ & $10(15.9)$ & $8(16)$ & $>0.9999$ \\
\hline \multicolumn{2}{|l|}{ CRS, n (\%) } & $79(69.9)$ & $37(58.7)$ & $42(84)$ & 0.0040 \\
\hline \multicolumn{2}{|l|}{ CRSwNP, n (\%) } & $54(47.8)$ & $27(42.9)$ & $27(54)$ & 0.0165 \\
\hline \multicolumn{2}{|l|}{ GERD, n (\%) } & $52(46)$ & $31(49.2)$ & $21(42)$ & 0.4554 \\
\hline \multirow[t]{4}{*}{ Chronic Mucus Hypersecretion, $\mathrm{n}$ (\%) } & & $44(38.9)$ & $15(23.8)$ & $29(58)$ & 0.0004 \\
\hline & Mucous & $7(6.2)$ & $4(6.4)$ & $3(6)$ & $>0.9999$ \\
\hline & Muco-purulent & $28(24.8)$ & $10(15.9)$ & $18(36)$ & 0.0167 \\
\hline & Purulent & $9(8)$ & I (I.6) & $8(16)$ & 0.0100 \\
\hline \multicolumn{2}{|l|}{ High dose ICS-LABA, n (\%) } & $113(100)$ & $63(100)$ & $50(100)$ & $>0.9999$ \\
\hline \multicolumn{2}{|l|}{ LAMA, n (\%) } & $78(69)$ & $43(68.3)$ & $35(70)$ & $>0.9999$ \\
\hline \multicolumn{2}{|l|}{ Patients on OCS, n (\%) } & $50(44.2)$ & $22(34.9)$ & $28(56)$ & 0.0357 \\
\hline \multicolumn{2}{|l|}{ Long-term macrolide therapy, n (\%) } & $14(12.4)$ & $3(4.8)$ & II (22) & 0.0084 \\
\hline \multicolumn{2}{|l|}{ Exacerbations/year, median (IQR) } & $8(4-12)$ & $6(4-12)$ & $10(4-12)$ & 0.0487 \\
\hline \multicolumn{2}{|l|}{ ACT, median (IQR) } & $13(9-16)$ & $13(10-16)$ & $12(9-16)$ & 0.9046 \\
\hline \multicolumn{2}{|l|}{$\mathrm{FEV}_{\mathrm{l}}, \mathrm{mL}$, mean $(\mathrm{SD})$} & $1969(720)$ & 1987 (687) & $1947(766)$ & 0.7700 \\
\hline \multicolumn{2}{|l|}{$\mathrm{FEV}_{1}$, \% predicted, mean (SD) } & $70.7(20.3)$ & $70.8(17.6)$ & $70.6(23.4)$ & 0.9461 \\
\hline \multicolumn{2}{|l|}{$\mathrm{FEV}_{\mathrm{I}} / \mathrm{FVC}, \%$, mean $(\mathrm{SD})$} & $64.5(14.2)$ & $63.3(14.3)$ & $66.1(14.1)$ & 0.3522 \\
\hline \multicolumn{2}{|l|}{ Total IgE, Ul/mL, median (IQR) } & $185(93-474)$ & I $77(87-40 \mid)$ & $223(95-559)$ & 0.6203 \\
\hline \multicolumn{2}{|c|}{$\begin{array}{l}\text { Eosinophil counts in peripheral blood, cells/ } \mu \mathrm{L} \text { median } \\
\text { (IQR) }\end{array}$} & $500(340-900)$ & $480(330-945)$ & $635(337-898)$ & 0.6102 \\
\hline \multicolumn{2}{|l|}{ Patients with $\geq 300$ eosinophils/ $\mu \mathrm{L}, \mathrm{n}$ (\%) } & $93(82.3)$ & $5 I(8 I)$ & $42(84)$ & 0.8054 \\
\hline \multicolumn{2}{|l|}{ FeNO, ppb, median (IQR) } & $34(17-56)$ & $34(15.5-54)$ & $34.5(18.5-67)$ & 0.7456 \\
\hline \multicolumn{2}{|l|}{ Vitamin D, ng/dL, median (IQR) } & 21 (16-29) & $21(17-29)$ & 21 (15-29) & $>0.9999$ \\
\hline \multicolumn{2}{|l|}{ Alpha-I antitrypsin, mg/dL, median (IQR) } & $152(130-170)$ & 151 (135-179) & $153(122-166)$ & 0.7980 \\
\hline
\end{tabular}

Notes: Categorical variables are presented as numbers (n) and percentages (\%). For normally distributed data, values are mean and standard deviation (SD). For nonnormally distributed variables, values are median and interquartile range (IQR). Bold entries highlight statistically significant differences between Type 2-High Severe Asthma (T2-SA) and Type 2-High Severe Asthma + Bronchiectasis (T2-SA+BE).

Abbreviations: ACT, asthma control test; BMI, body mass index; CRS, chronic rhinosinusitis; CRSwNP, chronic rhinosinusitis with nasal polyps; GERD, gastroesophageal reflux disease; LABA, long-acting beta agonist; ICS, inhaled corticosteroids; LAMA, long-acting muscarinic antagonist; FeNO, fractional exhaled nitric oxide; FEV, , forced expiratory volume in I second; FVC, forced vital capacity; IgE, immunoglobulin E. 
A

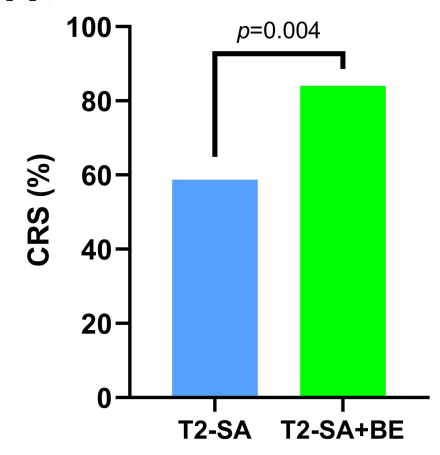

D

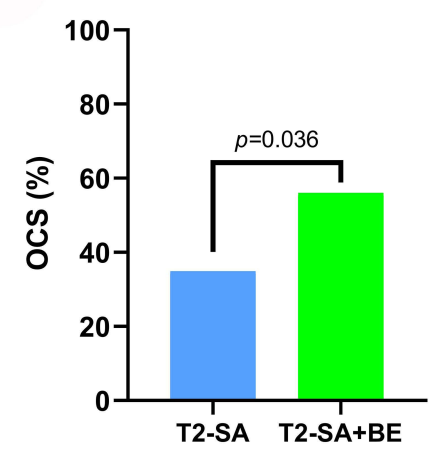

B

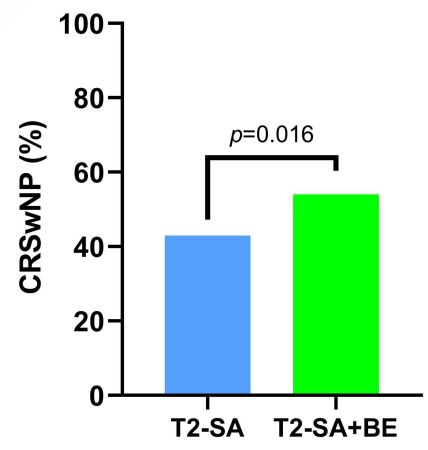

E

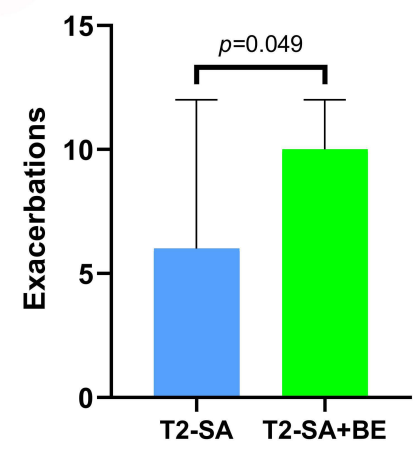

C

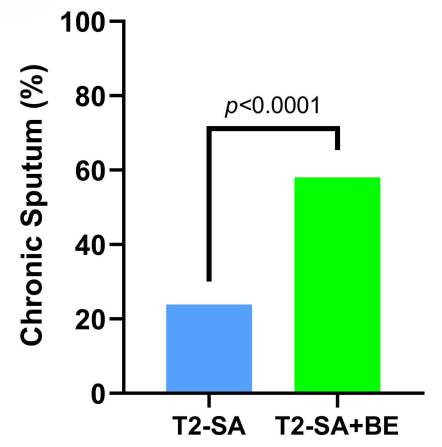

F

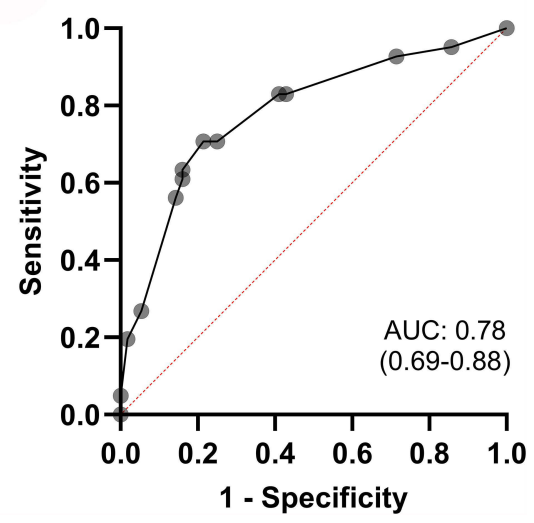

Figure 2 Differences between Type-2 High Severe Asthma patients with and without Bronchiectasis with regard to chronic rhinosinusitis (A), chronic rhinosinusitis with nasal polyps (B), chronic sputum production (C), chronic oral corticosteroids intake (D) and asthma exacerbations in the previous year (E). Receiver operating characteristics (ROC) of the optimal regression model of variables (presence of chronic rhinosinusitis, chronic mucus hypersecretion and daily OCS intake) strongly associated with comorbid BE in T2-SA (F).

Abbreviations: T2-SA, type 2-high severe asthma; BE, bronchiectasis; CRS, chronic rhinosinusitis; CRSwNP, chronic rhinosinusitis with nasal polyps; OCS, oral corticosteroids.

was $44.2 \%$, in line with the already published literature. ${ }^{11,32-35}$

Our results confirm and expand the current knowledge reporting that T2-SA with coexisting $\mathrm{BE}$ was frequently associated with CRS with and without nasal polyposis, chronic sputum production, chronic OCS intake, and a higher exacerbations rate. ${ }^{10}$

We hypothesized that the post-nasal drip, which is the hallmark symptom of CRS, together with an abundant eosinophilic bronchial inflammation, might play a role in $\mathrm{BE}$ development. Discharge of mucus from the upper to the lower airways could act as an irritative stimulus for the bronchial epithelium, promoting the recruitment of neutrophils, and in patients with type 2-high inflammation, may determine an exaggerated recall and activation of the eosinophils via the innate immunity pathway through IL-25,
IL-33 and TSLP production and ILC2 stimulation. Several studies have demonstrated that in patients with severe eosinophilic asthma and co-presence of CRS, particularly with nasal polyps, a greater degree of eosinophilic infiltration characterizes the bronchial mucosa; this feature makes the airways of patients affected by this combination of diseases more prone to eosinophilic inflammation. ${ }^{36,37}$ This pathogenic hypothesis is also corroborated by the already published literature data, which confirm this common association. ${ }^{36-39}$

Mucus hyperproduction is a clinical characteristic of both T2-SA and BE. ${ }^{40,41}$ The combination of these diseases may lead to a synergistic effect in sputum production. ${ }^{42,43}$ To this extent, almost $60 \%$ of our $\mathrm{T} 2-\mathrm{SA}+\mathrm{BE}$ patients reported $\mathrm{CMH}$, often purulent or muco-purulent. The persistent accumulation of mucus can limit airflow, worsen asthma 
Table 2 Radiological and Microbiological Features in Patients with $\mathrm{T} 2-\mathrm{SA}+\mathrm{BE}$

\begin{tabular}{|c|c|}
\hline \multicolumn{2}{|l|}{ T2-SA+BE, $n=50$} \\
\hline BHALLA score, median (IQR) & $18.3(16-20)$ \\
\hline BHALLA, $\mathbf{n}(\%)$ & \\
\hline Mild (16-25) & $38(76)$ \\
\hline Moderate $(9-15)$ & $12(24)$ \\
\hline Severe $(0-8)$ & $0(0)$ \\
\hline $\begin{array}{l}\text { Number of lobes with bronchiectasis, } \\
\text { median (IQR) }\end{array}$ & $3(2-4)$ \\
\hline Lobes involved, $\mathbf{n}(\%)$ & \\
\hline Lower lobes & $26(52)$ \\
\hline Upper lobes & $4(8)$ \\
\hline Lower and upper lobes & $10(20)$ \\
\hline Middle lobe & $4(8)$ \\
\hline Middle and lower lobes & $6(12)$ \\
\hline Bronchiectasis pattern distribution, $\mathrm{n}(\%)$ & \\
\hline Peripheral & $36(72)$ \\
\hline Central & $4(8)$ \\
\hline Mixed & $10(20)$ \\
\hline Types of bronchiectasis, n (\%) & \\
\hline Cylindrical & $48(96)$ \\
\hline Multiple types & $2(4)$ \\
\hline BSI, median (IQR) & $6(4-8)$ \\
\hline BSI, n (\%) & \\
\hline Mild (0-4) & II (22) \\
\hline Moderate $(5-8)$ & $33(66)$ \\
\hline Severe $(\geq 9)$ & $6(12)$ \\
\hline Sputum cultures, n (\%) & \\
\hline Negative & $44(88)$ \\
\hline Pseudomonas aeruginosa & $5(10)$ \\
\hline Stenotrophomonas maltophilia & I (2) \\
\hline
\end{tabular}

Notes: Categorical variables were presented as numbers (n) and percentages (\%). For non-normally distributed variables, values are median and interquartile range (IQR). Abbreviation: BSI, Bronchiectasis Severity Index.

symptoms, trigger asthma exacerbations, and reduce the asthma control with the standard inhaled high dose ICS + LABA, often making chronic OCS intake necessary. This can expose patients to OCS adverse events and reduce the ability to fight infections due to the suppression of the type 1 immune response. ${ }^{9,24}$ As we showed, a more significant proportion of $\mathrm{T} 2-\mathrm{SA}+\mathrm{BE}$ patients were OCS dependent $(56 \%)$ than those without BE $(35 \%)$.

In our multivariable logistic model, all the abovementioned clinical features predicted almost $80 \%$ of T2$\mathrm{SA}$ in association with $\mathrm{BE}$, highlighting the importance of these characteristics in suspecting the co-presence of both diseases to identify the subjects in which performing a chest-CT scan should be mandatory. Therefore, the diagnosis of $\mathrm{T} 2-\mathrm{SA}+\mathrm{BE}$ is fundamental in order to adopt the appropriate therapeutic strategies. ${ }^{40,44,45}$

The high-resolution chest-CT scan allowed us to define the prevalent pattern and the type of bronchiectasis. We reported mostly cylindrical BE (96\%), with a median involvement of 3 lobes, predominantly the lower ones and peripheral distribution. Lower lobes predominance of $\mathrm{BE}$ is common in the majority of aetiologies of non-cystic fibrosis $\mathrm{BE}$, reflecting the gravity-dependent retention of secretions. ${ }^{46}$ In terms of radiological severity, according to the Bhalla score, around $90 \%$ of patients presented a mild-tomoderate peribronchial thickening, $62 \%$ a mild-to-moderate mucus plugging, with almost no sacculations or abscesses, bullae, collapse and consolidation. Dunican et $\mathrm{al}^{44}$ showed that eosinophils via eosinophil peroxidase (EPO) promote mucus plug formation and that mucus plugs are a common finding in HR chest CT scans of SA patients, particularly in those with comorbid BE, as confirmed in our data.

To better evaluate the radiological and clinical findings in relation to the impact of BE on T2-SA, we assessed the BSI. Our results showed 6 as median BSI value, indicating an overall moderate severity (range 5-8), with $22 \%$ of subjects classified as mild and $66 \%$ moderate. We found an inversely proportional correlation between BSI, ACT and $\mathrm{FEV}_{1}$, confirming that the greater severity of $\mathrm{BE}$ resulted in a less stable disease. Therefore, it is essential to establish targeted treatment for both diseases to improve symptoms and other clinical outcomes. ${ }^{47,48}$

Data obtained from this prospective observational study confirm the hypothesis that a dangerous liaison closely links T2-SA and BE. ${ }^{10}$ The mechanism underlying the pathogenetic role of type 2 inflammation as a cause of $\mathrm{BE}$ has not been fully unveiled yet. Eosinophils in the bronchial tissue induce damage through degranulation and release of enzymes such as eosinophil-derived neurotoxin, eosinophil cationic protein, eosinophil peroxidase, and major basic protein. ${ }^{49,50}$ The resulting airway injury stimulates the bronchial epithelial cells to produce IL-25, IL-33 and TSLP, a cluster of cytokines known as "alarmins", which activate the ILC-2 to produce IL-5, key in eosinophil recruitment and activation, IL-4 and IL-13, responsible for mucus production and sub-epithelial fibrosis with airway remodelling. ${ }^{51,52}$ Recent studies have shown that anti-IL-5 or anti-IL-5R $\alpha$ monoclonal antibodies could be effective in controlling $\mathrm{T} 2$ inflammation in $\mathrm{T} 2-\mathrm{SA}+\mathrm{BE}$ patients, ${ }^{53-60}$ limiting eosinophils tissue 


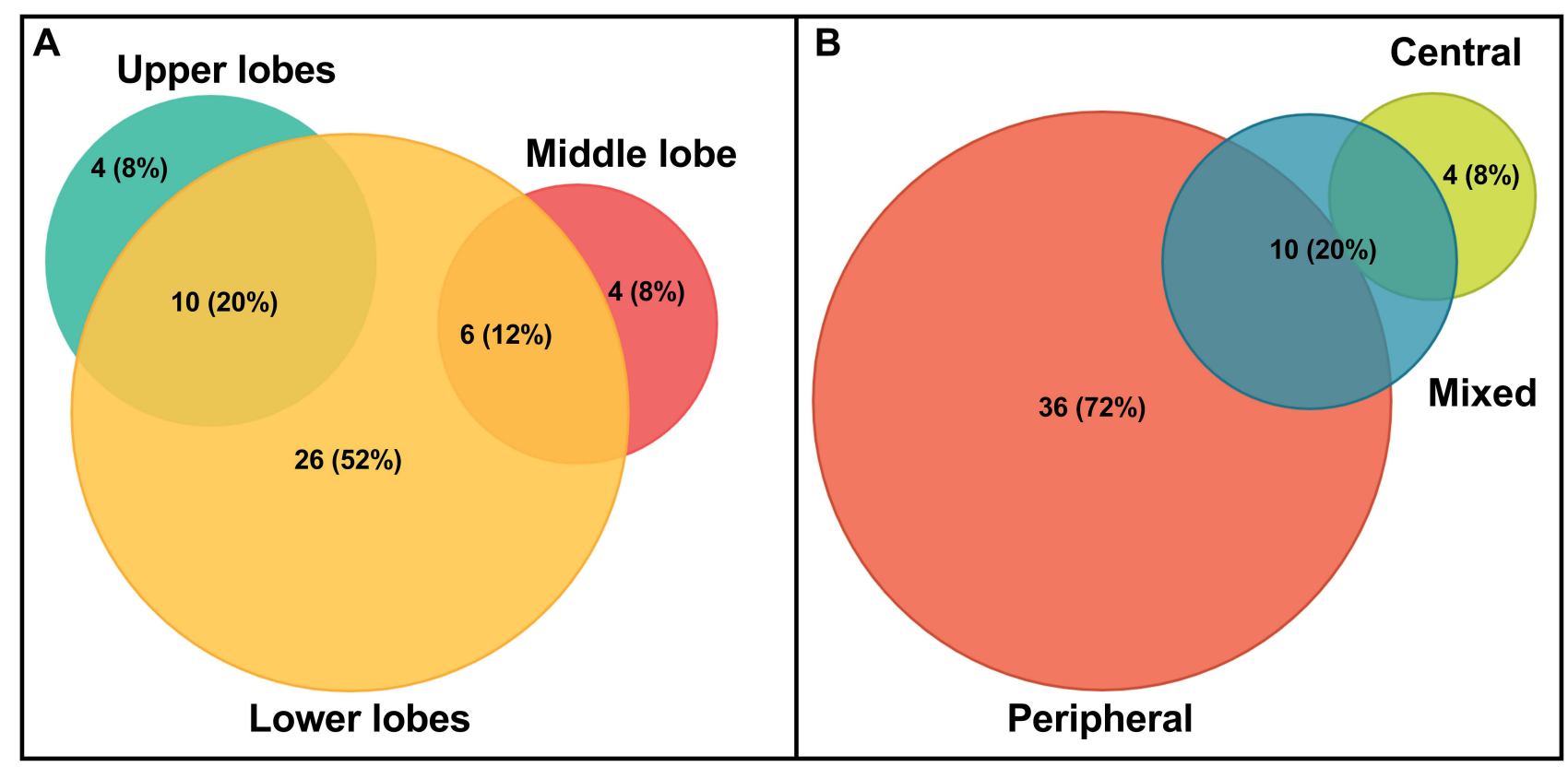

Figure 3 Bronchiectasis lobes involvement $(\mathbf{A})$ and distribution pattern (B).
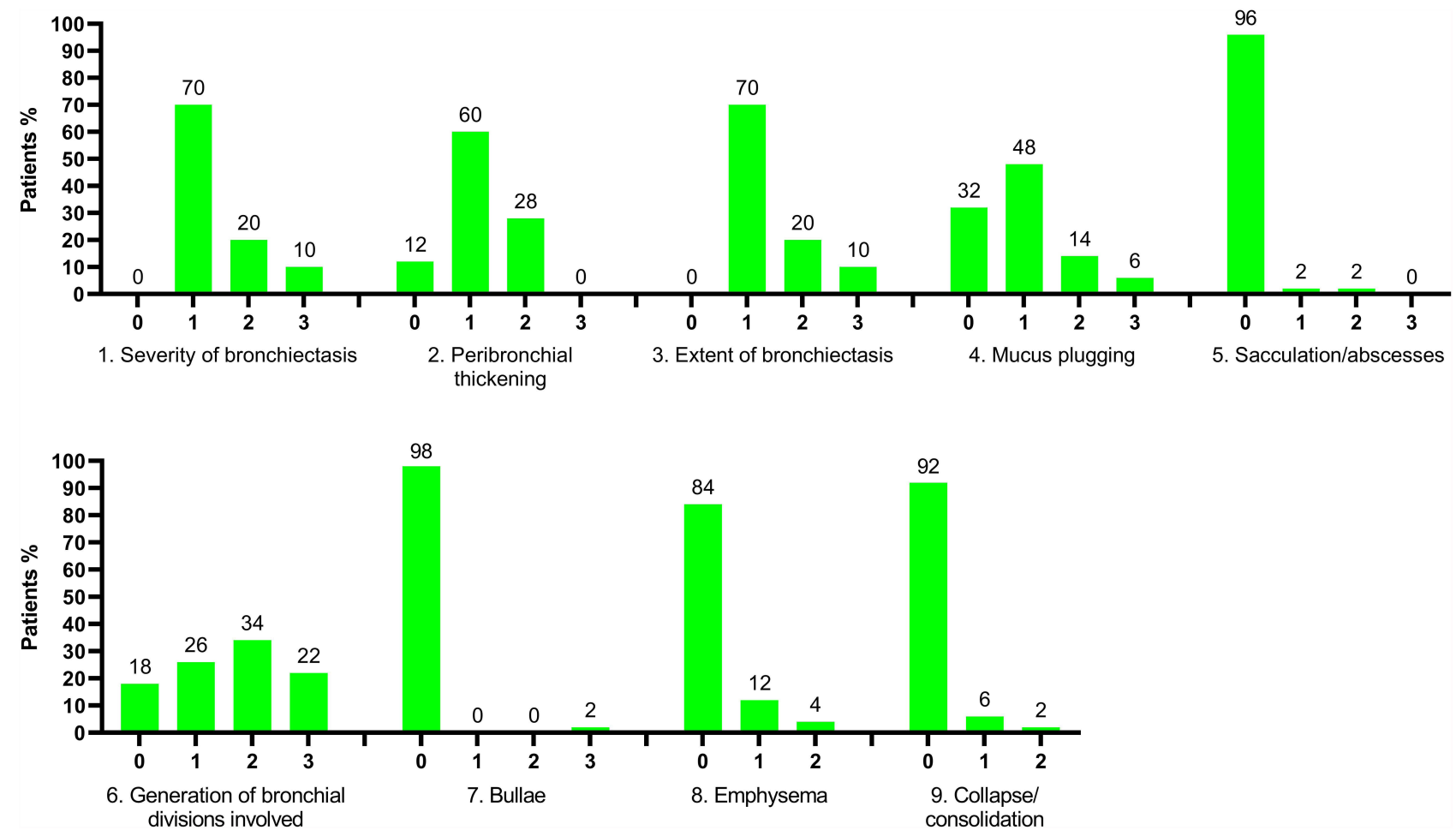

Figure 4 Distribution of patients in relation to Bhalla score items. Data as presented as percentage (\%). I. Severity of bronchiectasis: $0=$ None; I = luminal diameter slightly larger than the adjacent vessel; 2 = bronchial diameter between 2 and 3 times the diameter of the adjacent vessel; $3=$ bronchus is more than 3 times the diameter of the adjacent vessel. 2. Peribronchial thickening: $0=$ None; $I=$ wall thickness is similar to that of the surrounding vessel; $2=$ wall thickness greater, but less than twice, the diameter of the adjacent vessel; $3=$ more than twice the thickness of the adjacent vessel. 3 . Extent of bronchiectasis: $0=$ None; $I=I-5$ segments; $2=6-9 ; 3=>9.4$. Mucus plugs: $0=$ None; $I=1-5$ segments; $2=6-9 ; 3=>9$. 5 . Sacculation/abscesses: $0=$ None; $I=1-5$ segments; $2=6-9 ; 3=>9.6$. Generations of bronchial division involved: $0=$ None; $1=>4^{\mathrm{a}}$ generations; $2=>5^{\mathrm{a}} ; 3=>6^{\mathrm{a}}$. 7. Bullae: $0=$ None; $\mathrm{I}=$ unilateral; $2=$ bilateral; $3=>4$. 8 . Emphysema: $0=$ None; $I=1-5$ segments; $2=>5$. 9 . Collapse/ consolidation: 0 = None; $\mathrm{I}$ = subsegmental; 2 = segmental/lobar. 
A

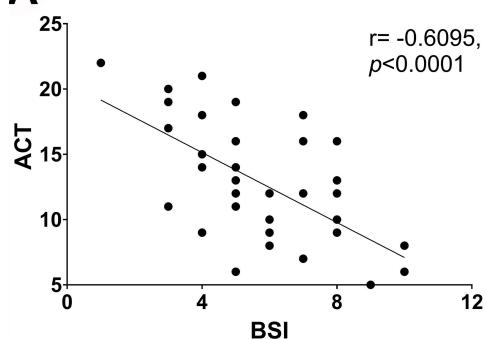

B

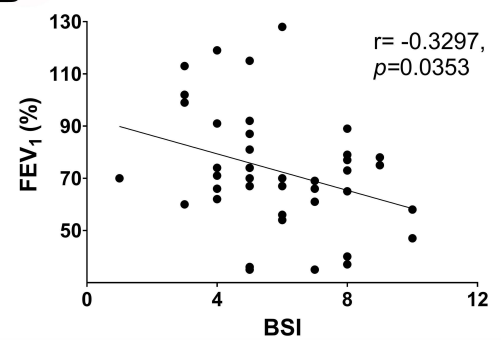

C

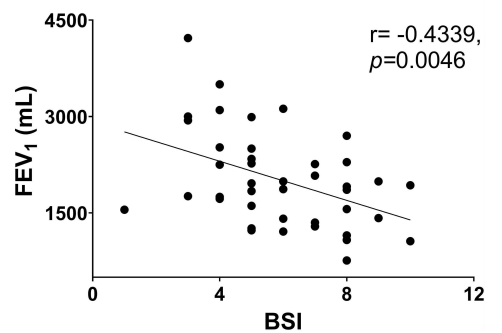

Figure 5 Correlations between BSI, ACT (A), FEV \% (B) and FEV $\mathrm{mL}(\mathbf{C})$.

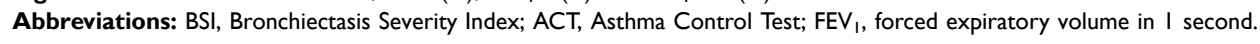

survival and reducing mucus stiffening and hypersecretion, with favourable outcomes concerning exacerbations, pulmonary function and asthma control, mainly when bronchiectasis severity was mild, reinforcing the importance of executing an early diagnosis of BE.

Tight junctions, which are the predominant structure that contribute to the epithelial barrier, act as gatekeepers to prevent the entry of microorganisms and foreign antigens. Tight junction breakdown has been suggested to have a causative role in asthma and bronchiectasis development. ${ }^{61}$ For this reason, T2-SA+BE patients likely present a marked alteration of this structure and consequently of the epithelial barrier and that could nurture a noxious loop of tissue inflammation and damage.

The main strength of this study is the prospective design and the exclusion of patients affected by other diseases often linked to BE. Therefore, we were able to evaluate and report for the first time the prevalence of BE in highly selected patients affected only by type 2-high SA. Furthermore, we used reliable tools, namely Bhalla and BSI, which allowed us to finely describe the radiological features, the clinical severity of BE and their impact on SA. Hence, the data collected are of high quality and harmonized, and findings coming from our study are robust and reliable.

We accurately excluded all the subjects with T2 low SA, cystic fibrosis, ABPA, low alpha-1 antitrypsin, immune deficiency, vasculitis and previous pneumonia episodes during the enrolment process, allowing us to characterize only those affected by genuine type 2-high SA, without other common confounding factors associated with BE.

The main limitations of the study are the absence of induced sputum and data on sputum cytology. Moreover, we did not perform any viral testing or routine sweat testing. Another important limitation, asthma exacerbations were assessed retrospectively, possibly hindering their correlation with BSI. Furthermore, this study could be underpowered to detect differences in outcomes, since its design did not consider a pre-specified sample size. Finally, all the patients were recruited only in two centres, limiting our external validity.

\section{Conclusion}

In conclusion, these results support the hypothesis that type-2 inflammation can have a causative role in the development of BE. Performing a HR chest CT scan is highly recommended when a diagnosis of T2-SA is made, especially in patients with CRS who report frequent asthma exacerbations, chronic sputum production and OCS dependency. The early detection of BE can be crucial to provide adequate treatment, necessary to improve and control patients' respiratory symptoms and quality of life.

\section{Abbreviations}

SA, severe asthma; T2-SA, type 2-high severe asthma; $\mathrm{BE}$, bronchiectasis; ACT, asthma control test; ABPA, allergic bronchopulmonary aspergillosis; CRSwNP, chronic rhinosinusitis with nasal polyps; $\mathrm{CMH}$, chronic mucus hypersecretion; BSI, Bronchiectasis Severity Index; BMI, body mass index; FeNO, fractional exhaled nitric oxide; OCS, oral corticosteroids (Prednisone); ICS, inhaled corticosteroids; LABA, long-acting beta agonist; LAMA, long-acting muscarinic antagonist; $\mathrm{FEV}_{1}$, forced expiratory volume in the 1st second; FVC, forced vital capacity; GERD, gastroesophageal reflux disease; IgE, immunoglobulin-E; IL-5, interleukin-5; IL-4, interleukin4; IL-13, interleukin-13; HRCT, high resolution computed tomography.

\section{Acknowledgment}

The statistical analysis was designed and performed in collaboration with Filippo Palermo, Professor of Biostatistics at the University of Catania. 


\section{Author Contributions}

All authors made substantial contributions to conception and design, acquisition of data, or analysis and interpretation of data, took part in drafting the article or revising it critically for important intellectual content, agreed to submit to the current journal, gave final approval of the version to be published, and agree to be accountable for all aspects of the work.

\section{Funding}

This research did not receive any specific grant from funding agencies in the public, commercial, or not-forprofit sectors.

\section{Disclosure}

Prof. Dr. Enrico Heffler reports personal fees from AstraZeneca, Sanofi, Regeneron, Novartis, GSK, Circassia, Stallergenes-Greer, and Nestlè Purina, outside the submitted work. The authors report no other potential conflicts of interest for this work.

\section{References}

1. Holguin F, Cardet JC, Chung KF, et al. Management of severe asthma: a European Respiratory Society/American Thoracic Society guideline. Eur Respir J. 2020;55(1):1900588. doi:10.1183/ 13993003.00588-2019

2. Chung KF, Wenzel SE, Brozek JL, et al. International ERS/ATS guidelines on definition, evaluation and treatment of severe asthma. Eur Respir J. 2014;43(2):343-373. doi:10.1183/09031936.00202013

3. Hekking PW, Wener RR, Amelink M, Zwinderman AH, Bouvy ML, Bel EH. The prevalence of severe refractory asthma. J Allergy Clin Immunol. 2015;135(4):896-902. doi:10.1016/j.jaci.2014.08.042

4. Global Initiative for Asthma (GINA). GINA Report, global strategy for asthma management and prevention; 2019. Available from: http:// ginasthma.org. Accessed February 05, 2021.

5. Melero Moreno C, Quirce S, Huerta A, Uría E, Cuesta M. Economic impact of severe asthma in Spain: multicentre observational longitudinal study. $J$ Asthma. 2019;56(8):861-871. doi:10.1080/ 02770903.2018.1499035

6. Kuruvilla ME, Lee FE, Lee GB. Understanding asthma phenotypes, endotypes, and mechanisms of disease. Clin Rev Allergy Immunol. 2019;56(2):219-233. doi:10.1007/s12016-018-8712-1

7. Bardin PG, Rangaswamy J, Yo SW. Managing comorbid conditions in severe asthma. Med J Aust. 2018;209(S2):S11-S17. doi:10.5694/ mja18.00196

8. Boulet LP, Boulay MÈ. Asthma-related comorbidities. Expert Rev Respir Med. 2011;5(3):377-393. doi:10.1586/ers.11.34

9. Polverino E, Dimakou K, Hurst J, et al. The overlap between bronchiectasis and chronic airway diseases: state of the art and future directions. Eur Respir J. 2018;52(3):1800328. doi:10.1183/ 13993003.00328-2018

10. Crimi C, Ferri S, Crimi N. Bronchiectasis and asthma: a dangerous liaison? Curr Opin Allergy Clin Immunol. 2019;19(1):46-52. doi:10.1097/ACI.0000000000000492

11. García-Clemente M, Enríquez-Rodríguez AI, Iscar-Urrutia M, et al. Severe asthma and bronchiectasis. J Asthma. 2020;57(5):505-509. doi:10.1080/02770903.2019.1579832
12. Polverino E, Goeminne PC, McDonnell MJ, et al. European respiratory society guidelines for the management of adult bronchiectasis. Eur Respir J. 2017;50(3):1700629. doi:10.1183/13993003.00629-2017

13. Crimi C, Ferri S, Campisi R, Crimi N. The link between asthma and bronchiectasis: state of the art. Respiration. 2020;99(6):463-476. doi: $10.1159 / 000507228$

14. Smith SG, Chen R, Kjarsgaard M, et al. Increased numbers of activated group 2 innate lymphoid cells in the airways of patients with severe asthma and persistent airway eosinophilia. J Allergy Clin Immunol. 2016;137(1):75-86.e8. doi:10.1016/j.jaci.2015.05.037

15. Choi Y, Sim S, Park HS. Distinct functions of eosinophils in severe asthma with type 2 phenotype: clinical implications. Korean J Intern Med. 2020;35(4):823-833. doi:10.3904/kjim.2020.022

16. Gramegna A, Aliberti S, Seia M, et al. When and how ruling out cystic fibrosis in adult patients with bronchiectasis. Multidiscip Respir Med. 2018;13(Suppl 1):29. doi:10.1186/s40248-018-0142-7

17. Reddel HK, Taylor DR, Bateman ED, et al. An official American Thoracic Society/European Respiratory Society statement: asthma control and exacerbations: standardizing endpoints for clinical asthma trials and clinical practice. Am J Respir Crit Care Med. 2009;180(1):59-99. doi:10.1164/rccm.200801-060ST

18. Miller MR, Hankinson J, Brusasco V, et al. Standardisation of spirometry. Eur Respir J. 2005;26(2):319-338. doi:10.1183/09031936.05.00034805

19. Nathan RA, Sorkness CA, Kosinski M, et al. Development of the asthma control test: a survey for assessing asthma control. J Allergy Clin Immunol. 2004;113(1):59-65. doi:10.1016/j.jaci.2003.09.008

20. Crimi C, Campisi R, Noto A, et al. Comparability of asthma control test scores between self and physician-administered test. Respir Med. 2020;170:106015. doi:10.1016/j.rmed.2020.10601

21. Dweik RA, Boggs PB, Erzurum SC, et al. An official ATS clinical practice guideline: interpretation of exhaled nitric oxide levels (FENO) for clinical applications. Am J Respir Crit Care Med. 2011;184(5):602-615. doi:10.1164/rccm.9120-11ST

22. Murray MP, Pentland JL, Turnbull K, MacQuarrie S, Hill AT. Sputum colour: a useful clinical tool in non-cystic fibrosis bronchiectasis. Eur Respir J. 2009;34(2):361-364. doi:10.1183/09031936.00163208

23. Fokkens WJ, Lund VJ, Hopkins C, et al. European position paper on rhinosinusitis and nasal polyps 2020. Rhinology. 2020;58 (SupplS29):1-464. doi:10.4193/Rhin20.600

24. Padilla-Galo A, Olveira C, Fernández de Rota-garcia L, et al. Factors associated with bronchiectasis in patients with uncontrolled asthma; the NOPES score: a study in 398 patients. Respir Res. 2018;19(1):43. doi:10.1186/s12931-018-0746-7

25. Lynch DA, Newell JD, Tschomper BA, Cink TM, Newman LS, Bethel R. Uncomplicated asthma in adults: comparison of CT appearance of the lungs in asthmatic and healthy subjects. Radiology. 1993;188(3):829-833. doi:10.1148/radiology.188.3.8351357

26. Naidich DP, McCauley DI, Khouri NF, Stitik FP, Siegelman SS. Computed tomography of bronchiectasis. J Comput Assist Tomogr. 1982;6(3):437-444. doi:10.1097/00004728-198206000-00001

27. Bhalla M, Turcios N, Aponte V, et al. Cystic fibrosis: scoring system with thin-section CT. Radiology. 1991;179(3):783-788. doi:10.1148/ radiology.179.3.2027992

28. Lm REID. Reduction in bronchial subdivision in bronchiectasis. Thorax. 1950;5(3):233-247. doi:10.1136/thx.5.3.233

29. Pasteur MC, Helliwell SM, Houghton SJ, et al. An investigation into causative factors in patients with bronchiectasis. Am J Respir Crit Care Med. 2000;162(4 Pt 1):1277-1284. doi:10.1164/ajrccm.162.4.9906120

30. Kwok WC, Ho JCM, Tam TCC, et al. Risk factors for Pseudomonas aeruginosa colonization in non-cystic fibrosis bronchiectasis and clinical implications. Respir Res. 2021;22(132). doi:10.1186/ s12931-021-01729-5

31. Chalmers JD, Goeminne P, Aliberti S, et al. The bronchiectasis severity index. An international derivation and validation study. $A m$ $J$ Respir Crit Care Med. 2014;189(5):576-585. doi:10.1164/ rccm.201309-1575OC 
32. Dimakou K, Gousiou A, Toumbis M, et al. Investigation of bronchiectasis in severe uncontrolled asthma. Clin Respir J. 2018;12 (3):1212-1218. doi:10.1111/crj.12653

33. Ferri S, Crimi C, Campisi R, et al. Impact of asthma on bronchiectasis severity and risk of exacerbations. J Asthma. 2020:1-12. doi: 10.1080/02770903.2020.1857395.

34. Perez-Miranda J, Traversi L, Polverino E. Bronchiectasis in severe asthma: a distinct phenotype? Curr Opin Pulm Med. 2019;25 (1):71-78. doi:10.1097/MCP.0000000000000542

35. Coman I, Pola-Bibián B, Barranco P, et al. Bronchiectasis in severe asthma: clinical features and outcomes. Ann Allergy Asthma Immunol. 2018;120(4):409-413. doi:10.1016/j.anai.2018.02.016

36. Handley E, Nicolson CH, Hew M, Lee AL. Prevalence and clinical implications of chronic rhinosinusitis in people with bronchiectasis: a systematic review. J Allergy Clin Immunol Pract. 2019;7(6):20042012.e1. doi:10.1016/j.jaip.2019.02.026

37. Canonica GW, Malvezzi L, Blasi F, et al. Chronic rhinosinusitis with nasal polyps impact in severe asthma patients: evidences from the Severe Asthma Network Italy (SANI) registry. Respir Med. 2020;166:105947. doi:10.1016/j.rmed.2020.105947

38. Martínez-Rivera C, Crespo A, Pinedo-Sierra C, et al. Mucus hypersecretion in asthma is associated with rhinosinusitis, polyps and exacerbations. Respir Med. 2018;135:22-28. doi:10.1016/j.rmed.2017.12.013

39. Peters AT, Bose S, Guo A, et al. Prevalence of bronchiectasis in patients with chronic rhinosinusitis in a tertiary care center. $J$ Allergy Clin Immunol Pract. 2021;9(8):3188-3195.e2. doi:10.1016/j.jaip.2021.04.054

40. Boucher RC. Muco-Obstructive Lung Diseases. $N$ Engl J Med. 2019;380(20):1941-1953. doi:10.1056/NEJMra1813799

41. Fanta $\mathrm{CH}$. Clinical aspects of mucus and mucous plugging in asthma. J Asthma. 1985;22(6):295-301. doi:10.3109/02770908509087113

42. Hammad H, Lambrecht BN. The basic immunology of asthma. Cell. 2021;184(6):1469-1485. doi:10.1016/j.cell.2021.02.016

43. Crespo-Lessmann A, Bernal S, Del Río E, et al. Association of the CFTR gene with asthma and airway mucus hypersecretion. PLoS One. 2021;16(6):e0251881. doi:10.1371/journal.pone.0251881

44. Dunican EM, Elicker BM, Gierada DS, et al. Mucus plugs in patients with asthma linked to eosinophilia and airflow obstruction. J Clin Invest. 2018;128(3):997-1009. doi:10.1172/JCI95693

45. Hoshino M, Matsuoka S, Handa H, Miyazawa T, Yagihashi K. Correlation between airflow limitation and airway dimensions assessed by multidetector CT in asthma. Respir Med. 2010;104 (6):794-800. doi:10.1016/j.rmed.2009.12.005

46. King PT, Holdsworth SR, Freezer NJ, Villanueva E, Holmes PW. Characterisation of the onset and presenting clinical features of adult bronchiectasis. Respir Med. 2006;100(12):2183-2189. doi:10.1016/j. rmed.2006.03.012

47. Crimi C, Campisi R, Cacopardo G, et al. Real-life effectiveness of mepolizumab in patients with severe refractory eosinophilic asthma and multiple comorbidities. World Allergy Organ J. 2020;13 (9):100462. doi:10.1016/j.waojou.2020.100462
48. Crimi C, Campisi R, Nolasco S, et al. Mepolizumab effectiveness in patients with severe eosinophilic asthma and co-presence of bronchiectasis: a real-world retrospective pilot study. Respir Med. 2021;185:106491. doi:10.1016/j.rmed.2021.106491

49. Henderson WR, Chi EY, Klebanoff SJ. Eosinophil peroxidase-induced mast cell secretion. J Exp Med. 1980;152 (2):265-279. doi:10.1084/jem.152.2.265

50. Carr TF, Berdnikovs S, Simon HU, Bochner BS, Rosenwasser LJ. Eosinophilic bioactivities in severe asthma. World Allergy Organ J. 2016;9:21. doi:10.1186/s40413-016-0112-5

51. Doherty T, Broide D. Cytokines and growth factors in airway remodeling in asthma. Curr Opin Immunol. 2007;19(6):676-680. doi:10.1016/j.coi.2007.07.017

52. Cho JY. Recent advances in mechanisms and treatments of airway remodeling in asthma: a message from the bench side to the clinic. Korean J Intern Med. 2011;26(4):367-383. doi:10.3904/kjim.2011.26.4.367

53. Coverstone AM, Seibold MA, Peters MC. Diagnosis and management of T2-high asthma. J Allergy Clin Immunol Pract. 2020;8 (2):442-450. doi:10.1016/j.jaip.2019.11.020

54. Kudlaty E, Patel GB, Prickett ML, Yeh C, Peters AT. Efficacy of type 2-targeted biologics in patients with asthma and bronchiectasis. Ann Allergy Asthma Immunol. 2021;126(3):302-304. doi:10.1016/j. anai.2020.11.014

55. Pelaia C, Crimi C, Vatrella A, Tinello C, Terracciano R, Pelaia G. Molecular Targets for Biological Therapies of Severe Asthma. Front Immunol. 2020;11:603312. doi:10.3389/fimmu.2020.603312

56. Nolasco S, Crimi C, Pelaia C, et al. Benralizumab effectiveness in severe eosinophilic asthma with and without chronic rhinosinusitis with nasal polyps: a Real-World Multicenter Study. J Allergy Clin Immunol Pract. 2021:S2213-2198(21) 00903-X. doi: 10.1016/j. jaip.2021.08.004.

57. Carpagnano GE, Scioscia G, Lacedonia D, Curradi G, Foschino Barbaro MP. Severe uncontrolled asthma with bronchiectasis: a pilot study of an emerging phenotype that responds to mepolizumab. J Asthma Allergy. 2019;12:83-90. doi:10.2147/JAA.S196200

58. Ferri S, Crimi C, Heffler E, Campisi R, Noto A, Crimi N. Vitamin D and disease severity in bronchiectasis. Respir Med. 2019;148:1-5. doi:10.1016/j.rmed.2019.01.009

59. Pelaia C, Crimi C, Benfante A, et al. Therapeutic effects of benralizumab assessed in patients with severe eosinophilic asthma: real-life evaluation correlated with allergic and non-allergic phenotype expression. J Asthma Allergy. 2021;14:163-173. doi:10.2147/JAA.S297273

60. Nolasco S, Campisi R, Intravaia R, et al. Case Report: acute effect of benralizumab on asthma exacerbation without concomitant corticosteroid use. F1000Res. 2020;9:637. doi:10.12688/f1000research.24603.2

61. Sugita K, Kabashima K. Tight junctions in the development of asthma, chronic rhinosinusitis, atopic dermatitis, eosinophilic esophagitis, and inflammatory bowel diseases. J Leukoc Biol. 2020;107 (5):749-762. doi:10.1002/JLB.5MR0120-230R

Journal of Asthma and Allergy

\section{Dovepress}

\section{Publish your work in this journal}

The Journal of Asthma and Allergy is an international, peer-reviewed open-access journal publishing original research, reports, editorials and commentaries on the following topics: Asthma; Pulmonary physiology; Asthma related clinical health; Clinical immunology and the immunological basis of disease; Pharmacological interventions and new therapies. The manuscript management system is completely online and includes a very quick and fair peer-review system, which is all easy to use. Visit http://www.dovepress.com/testimonials.php to read real quotes from published authors. 